\title{
The Polymerization of Furfuryl Alcohol with p-toluenesulfonic Acid: Photocross- Linkeable Feature of the Polymer
}

\author{
Martha Principe, Ricardo Martínez, Pedro Ortiz and Jacques Rieumont \\ IMRE, Faculdad Química, Universidad de La Habana
}

\begin{abstract}
Poly(furfuryl alcohol) with different amounts of oxymethylenic bridges was synthesized using trifluoroacetic and p-toluenesulfonic acid. All polymers displayed a tendency to retain acids. The isolated products containing traces of acid became insoluble in a few hours; while neutral material maintains their solubility for at least one month. Polymers stored in solution were stable according to their ${ }^{1} \mathrm{H}$ NMR spectra. Polymers cross-linked after being exposed to UV radiation. The product of the reaction of polymer with maleic anhydride is useful for preparing negative photoresists.
\end{abstract}

Keywords: Furfuryl alcohol, polymerization, cross-link, photoresist.

Introduction.

Furfuryl alcohol (FA) polymerizes by Brönsted and Lewis acid yielding colored polymers with rather complex structures ${ }^{[1]}$. The study of the polymerization of FA with trifluoroacetic acid, conducted in methylene dichloride, shows that the number of chains with ether bonds depends on the ratio of the initial concentrations of the monomer to the acid and oxymethylenic bridges can be reduced to zero ${ }^{[2,3]}$. However, changes in experimental conditions do not avoid the formation of branched sequences and the buildup of color, which indicates that both phenomena are inherent to the mechanism of polymerization.

The easy cross-linking of resins from FA by acids and heat have found applications in a wide variety of technologies such as metal-casting cores and molds; corrosion-resistant materials; polymer concretes and others ${ }^{[4]}$.

Color has been considered one of the disadvantages of these resins. Small amounts of very strong chromophores are present along the chains; their concentrations are too low to be detected by spectroscopy. Recently, the mechanism of the origin of color was explained with the help of model systems ${ }^{[5]}$. Polyunsaturated sequences are produced

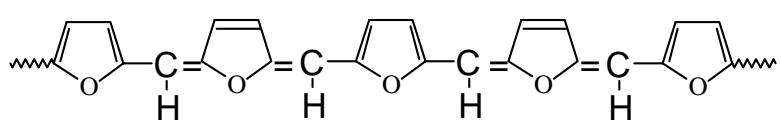

by successive hydride-ion/proton abstractions from methylene groups.

This work is aimed at taking advantage of the presence of polyunsaturated sequences in the resins from FA through photocross-linking by UV light and the reported tendency of the polymer to become insoluble in presence of air ${ }^{[2]}$. Hence, we also studied the influence of the neutralization of the polymerizing solution on polymer solubility as well as the aging of the polymer solution whether containing traces of acid or not.

\section{Materials and Experimental Procedures.}

Materials: The purification of furfuryl alcohol (FA, from Merck) has been reported elsewhere ${ }^{[2]}$.

Methylene dichloride (DCM, from BDH), trifluoroacetic acid (TFA, from Carlo Erba), tetrahydrofuran (THF), p-toluenesulphonic acid (PTS) and other reagents (all from BDH) were used in the procedures without further purification.

Techniques: Polymerization was carried out in a threeneck flask, under reflux and stirring (Table 1). Solid

Autor para correspôndência: Ricardo Martínez, IMRE, Faculdad Química, Universidad de La Habana, Habana 10400, CUBA, e-mail: ricardo@fq.oc.uh.cu 
PTS was added slowly into the FA solution to insure homogeneity of the polymerizing solution. Acid solution was dripped into the monomer solution when TFA was used as the initiator. The neutralization of the polymerizing solution with a concentrated basic solution may easily lead to emulsification. The use of a $1 \mathrm{M} \mathrm{NaOH}$ solution requires two washes of 10 minutes each but at the end of the reaction an emulsion may appear. In order to avoid this problem an excess of $0.1 \mathrm{M} \mathrm{NaOH}$ solution was used.

The neutralization of the reaction mixture was also followed by titration. An excess of a base solution $\left(\mathrm{KOH}\right.$ or $\left.\mathrm{Na}_{2} \mathrm{CO}_{3}\right) 0.1 \mathrm{M}$ was added to the sample and stirred for 10 minutes. Afterwards, the aqueous layer was removed and titrated with $0.1 \mathrm{M}$ $\mathrm{HCl}$ solution. This process was repeated until the polymer solution became neutralized.

Polymers were isolated by precipitation in petroleum ether and dried by using a high vacuum line.

Diels-Alders adduct. PolyFA and maleic anhydride $(10: 1 \mathrm{~g} / \mathrm{g})$ were dissolved in THF and heated at $40^{\circ} \mathrm{C}$ for 3 hours. The product was isolated by precipitation in petroleum ether.

Acrylic copolymer from methyl methacrylate, butyl methacrylate and methacrylic acid (molar composition 42:49:9) was supplied by R. Lago ${ }^{[6]}$.

Photoresists were prepared by dissolving the adduct and the acrylic copolymer $(10: 1 \mathrm{~g} / \mathrm{g})$ in chloroform. Films were formed over $\mathrm{Cu}$ plate or flat silicon by immersion or spin coating, respectively, followed by evaporation of the solvent at $50^{\circ} \mathrm{C}$. The resists were exposed to the UV light from a Hg lamp $(200 \mathrm{~W})$. The negative patterns were developed by treating the resists with a mixture of toluene, trichloroethylene and chloroform (1:2:7).

Measurements: NMR spectra were recorded using a Bruker AMX-600 spectrometer equipped with an ASPECT 300 computer, a the frequencies of $600 \mathrm{Mhz}\left({ }^{1} \mathrm{H}\right)$ and $150 \mathrm{Mhz}\left({ }^{13} \mathrm{C}\right)$. The polymers were dissolved in $\mathrm{CDCl}_{3}$ and the temperature were regulated to $300 \mathrm{~K}$. The nature of each carbon atom was determined by using the distortionless enhancement by polarization transfer technique (DEPT). with proton pulses at $135^{\circ}$ and $90^{\circ}$ respectively.

Number-average molecular weights, were determined by vapor-pressure osmometry using a Knauer equipment.

\section{Results and Discussion}

\section{Polymer solubility and neutralization of the polymerizing solution.}

Table 1 shows representative and reproducible results from the experiments carried out in this work. According to Gonzalez et al. ${ }^{[2]}$ polymer chains that are produced by polymerization with $[\mathrm{FA}]_{0} /[\mathrm{TFA}]_{0}=$ 20 should have few oxymethylenic bridges. That was confirmed with Polymer P1, which was synthesized as a reference. However, with PTS the polymer (P2) is free of ether bridges and a relation $[\mathrm{FA}]_{0} /[\mathrm{PTS}]_{0}>$ 20 is required to obtain this sequence (P3 and P4).

PTS is insoluble in DCM but with the addition of FA the system becomes homogeneous. The polymerization with PTS is faster than with TFA and reticulation is reached in experiments of series $\mathrm{P} 2$ with [FA]/ $[$ acid $]=20$. Approximately, $34-40 \%$ of the polymer is insoluble.

Different behavior in the solubility of the isolated polymer depending on the percentage of neutralization of the polymerizing solution and the presence of air is observed. Polymers arising from a poorly neutralized solution became insoluble approximately two hours after being isolated and dried; while, polymers obtained after a good neutralization (higher than $90 \%$ ) kept their solubility for at least one month. However, the exclusion of air by drying the polyFA in a high vacuum line avoid the reticulation of both kinds of samples. These results indicate that the polymer becomes insoluble by oxidation but this process is accelerated by acids.

Table 1. Polymerization of furfuryl alcohol with p-toluenesulfonic acid and trifluoroacetic acid

\begin{tabular}{|c|c|c|c|c|c|c|c|}
\hline Polymer & Acid & {$[\mathrm{FA}]_{0} /[\text { Acid }]_{0}$} & $\begin{array}{l}\text { Acid] }_{0} \\
\text { mol.L }\end{array}$ & $\begin{array}{c}\mathrm{T} \\
\left({ }^{\circ} \mathbf{C}\right)\end{array}$ & $\begin{array}{c}\text { t } \\
\text { (h) }\end{array}$ & $\begin{array}{c}\% \text { of } \\
\text { neutralization }\end{array}$ & $\begin{array}{c}\overline{M n} \\
\text { g/mol }\end{array}$ \\
\hline $\mathrm{P} 1$ & TFA & 20 & 0.2 & 40 & 10 & 90 & 2400 \\
\hline $\mathrm{P} 2$ & PTS & 20 & 0.2 & 40 & 10 & 91 & 2155 \\
\hline P3 & PTS & 70 & 0.1 & 20 & 24 & 90 & 3200 \\
\hline $\mathrm{P} 4$ & PTS & 70 & 0.1 & 20 & 24 & 60 & 3522 \\
\hline
\end{tabular}




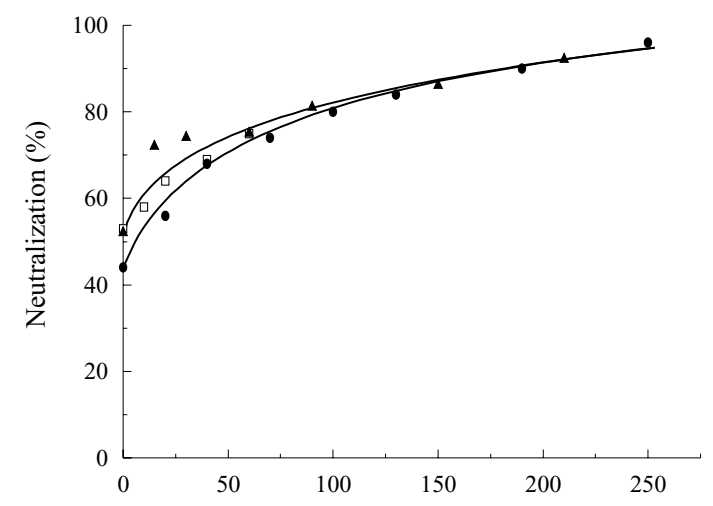

Figure 1. Time dependence of neuttrimiation of $\mathrm{P}_{1}$ (Table 1) $\square \mathrm{KOH}$ $0.1 \mathrm{M}$ was used for neutralization; $\bullet$ Polymer sample was extracted with water, neutralized with an excess of $\mathrm{KOH} 0.1 \mathrm{M}$ and titrated with $\mathrm{HCl} 0.1 \mathrm{M} ; \boldsymbol{\Delta} \mathrm{Na}_{2} \mathrm{CO}_{3} 0.05 \mathrm{M}$ was used for neutralization

PolyFA has the capacity to retain acids. Figure 1 shows that the neutralization of the polymerizing solution is slow and that not all of the acid is free. After the first base addition only about $50 \%$ of the acid is titrated. The first extraction with basic solutions (represented with triangles and squares) was somewhat more efficient than with water (represented with circles). However, the neutralization curves over $70 \%$ tended to be identical. This phenomenon is analogous to what was observed in the polymerization of 2-propenylfuran where around 30\% of the initial amount of the acid was retained by the polymer probably in the form of esters ${ }^{[7]}$ and in the polymerization of 2-vinylfuran where the consumption of acid is higher $(\approx 70 \%)$ due to the additional and more important formation of conjugated ions ${ }^{[8]}$. Both the pres- ence of esters ${ }^{[3]}$ and conjugated ions ${ }^{[5]}$ in the polymerization of FA were reported and are in accordance with acid consumption.

\section{Aging of the polymer solution}

Both good and poorly neutralized solutions of polyFA were examined with the help of NMR spectroscopy.

Polymer structures were determined by ${ }^{1} \mathrm{H}$ and ${ }^{13} \mathrm{C}$ NMR. The assignment of signals can be found elsewhere ${ }^{[2,9-11]}$. Scheme 1 shows the three main structures that occur depending on the reaction conditions as well as some significant signals ( $\square$ values in ppm) of their ${ }^{1} \mathrm{H}$ and ${ }^{13} \mathrm{C}$ NMR spectra:
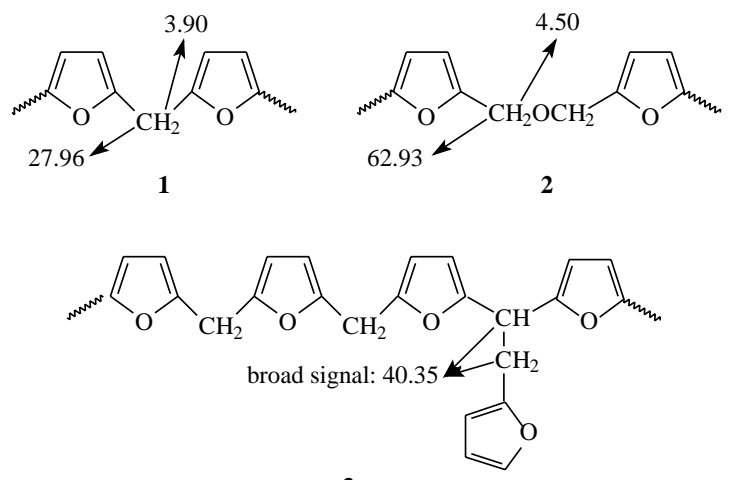

Scheme 1

Examples of polymer spectra are shown in Figures 2 and 3.

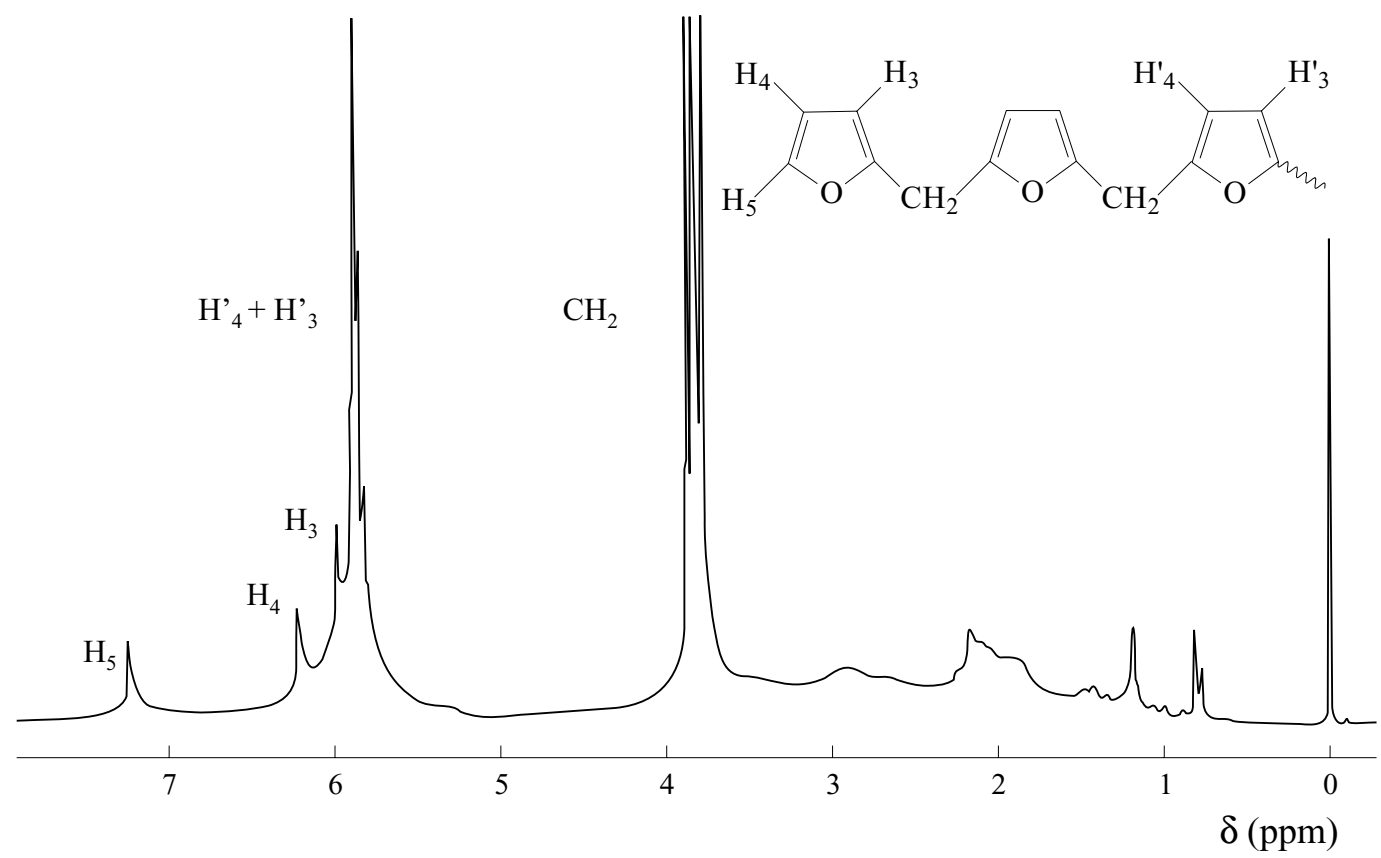

Figure 2. ${ }^{1} \mathrm{H}$ NMR spectrum $(600 \mathrm{MHz})$ of polyfurfuryl alcohol without ether bridges 


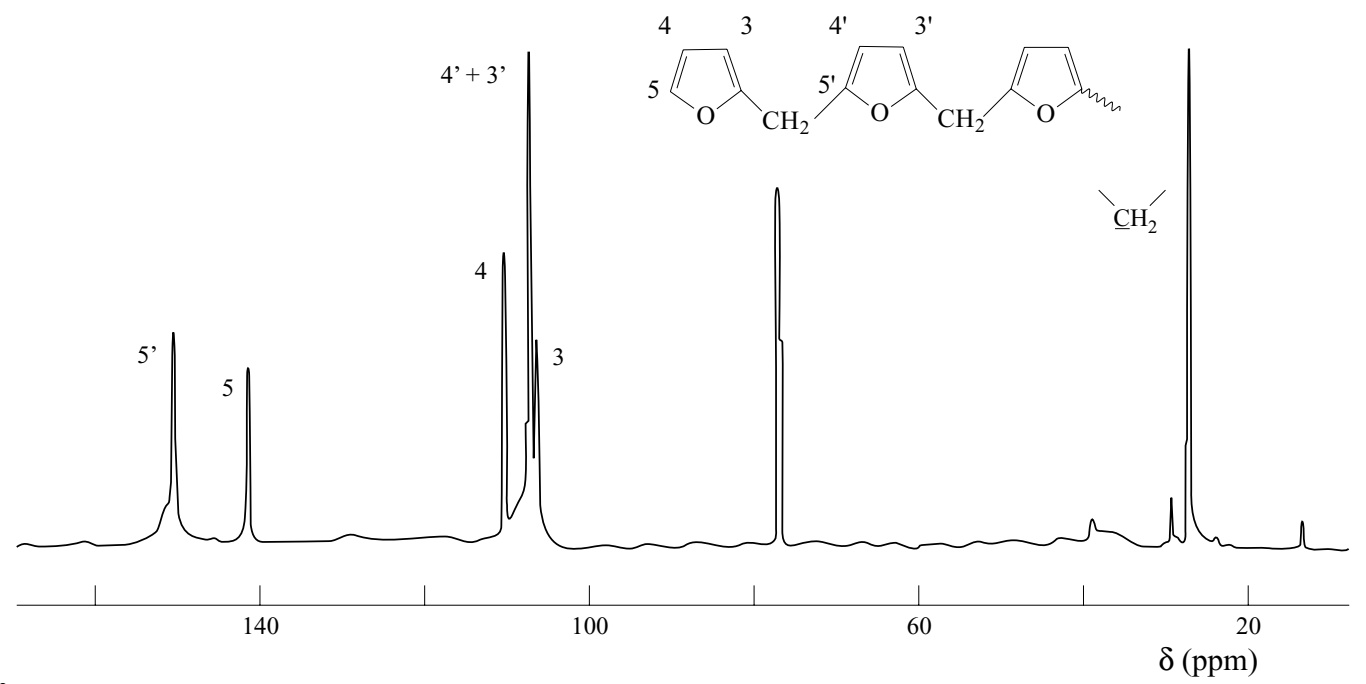

Figure 3. ${ }^{13} \mathrm{C}$ NMR spectrum $(150 \mathrm{MHz})$ of polyfurfuryl alcohol without ether bridges

No signals are observed around $\delta=4.50 \mathrm{ppm}$ in the ${ }^{1} \mathrm{H}$ NMR spectrum $(600 \mathrm{MHz})$ of $\mathrm{P} 1$ (Figure 2) indicating the absence of $\mathrm{CH}_{2}-\mathrm{O}-\mathrm{CH}_{2}$ bridges in the polymer. Figure 3 exhibits the ${ }^{13} \mathrm{C}$ NMR $(150 \mathrm{MHz})$ with the assignment of the signals supported by a determination applying DEPT technique. The signals at $\delta=29.29$ and 38.83 ppm suggests that the branched sequence 3 accompanies the main sequence 1 of polyFA.

The ${ }^{1} \mathrm{H}$ and ${ }^{13} \mathrm{C}$ NMR spectra of the polymers prepared with $[\mathrm{FA}] /[\mathrm{PTS}]=70$ exhibit intense signals at $\delta=4.50$ and 62.93 , respectively, which confirm the presence of significant $\mathrm{CH}_{2}-\mathrm{O}-\mathrm{CH}_{2}$ bridges in the polymer.

The ${ }^{1} \mathrm{H}$ NMR spectra of the polymers reported in Table 1 were recorded for a month. Some selected proton intensity ratios were chosen as the criterion for structural changes, namely:

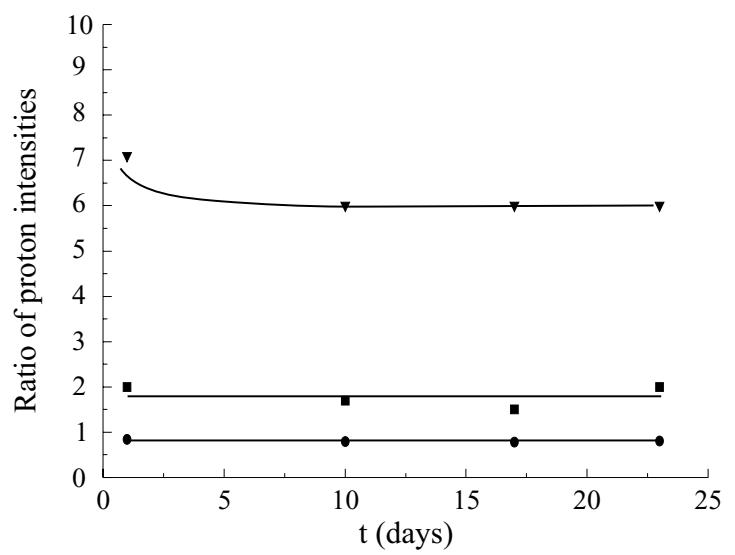

Figure 4. Time dependence of some ratios of proton intensities of ${ }^{1} \mathrm{H}$ NMR spectra of polyfurfuryl alcohol $\left(\mathrm{P}_{2}\right.$, Table 1): $\mathbf{\nabla} \mathrm{I} \mathrm{C}_{2} / \mathrm{I} \mathrm{H}_{5}$;

- I $\Sigma \mathrm{H}_{\text {Furan }} / \mathrm{I} \mathrm{CH}_{2} ; \bullet\left(\mathrm{I}^{2} \mathrm{H}_{(3+4)}-2 \mathrm{I} \mathrm{H}_{5}\right) / \mathrm{I} \Sigma \mathrm{H}_{(3+4)}$

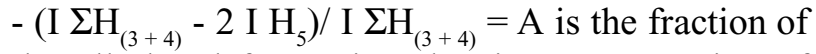
the alkylated furan ring that is representative of sequence 3 (scheme 1)

- I $\underline{\mathrm{C}}_{2} / \mathrm{I} \mathrm{H}_{5}=\mathrm{X}_{\mathrm{n}}$ if only linear sequence 1 was present.

- I $\Sigma \mathrm{H}_{\text {Furan }} / \mathrm{I} \mathrm{C}_{2}=\mathrm{MB}$. This relation is proportional to the amount of methylene bridges in the polymer while

- I $\Sigma \mathrm{H}_{\text {Furan }} / \mathrm{I} \mathrm{C} \underline{H}_{2}-\mathrm{O}-\underline{\mathrm{CH}}_{2}=\mathrm{EB}$ is proportional to the number of ether bridges.

Figure 4 shows almost no change in these ratios for a polymer of series $\mathrm{P} 2$. The same behavior was observed in the other polymers. Spectra were virtually unchanged and the average of the reference ratios measured during one month are shown in Table 2.

However, when some FA remained in a poorly neutralized sample the spectra differed over time due to additional, but slow polymerization.

Table 2. Mean values of some selected ratios of proton intensities from ${ }^{1} \mathrm{H}$ NMR spectra of polyFA samples measured during one month

\begin{tabular}{ccccc}
\hline Intensity ratios & \multicolumn{4}{c}{ Samples } \\
\cline { 2 - 5 } & P1 & P2 & P3 & P4 \\
\hline A x 100 & 76 & 80 & 82 & 70 \\
MB & 1.5 & 1.8 & 1.7 & 1.8 \\
EB & 3.9 & - & 2.5 & 2.6 \\
$\mathrm{X}_{\mathrm{n}}$ & 6.4 & 6.4 & 7.4 & 4.2 \\
\hline
\end{tabular}

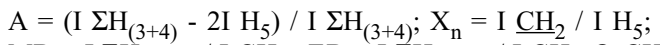

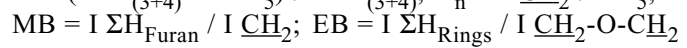


<smiles>Cc1oc(CO)c(C)c1C</smiles>

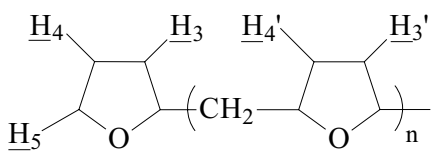

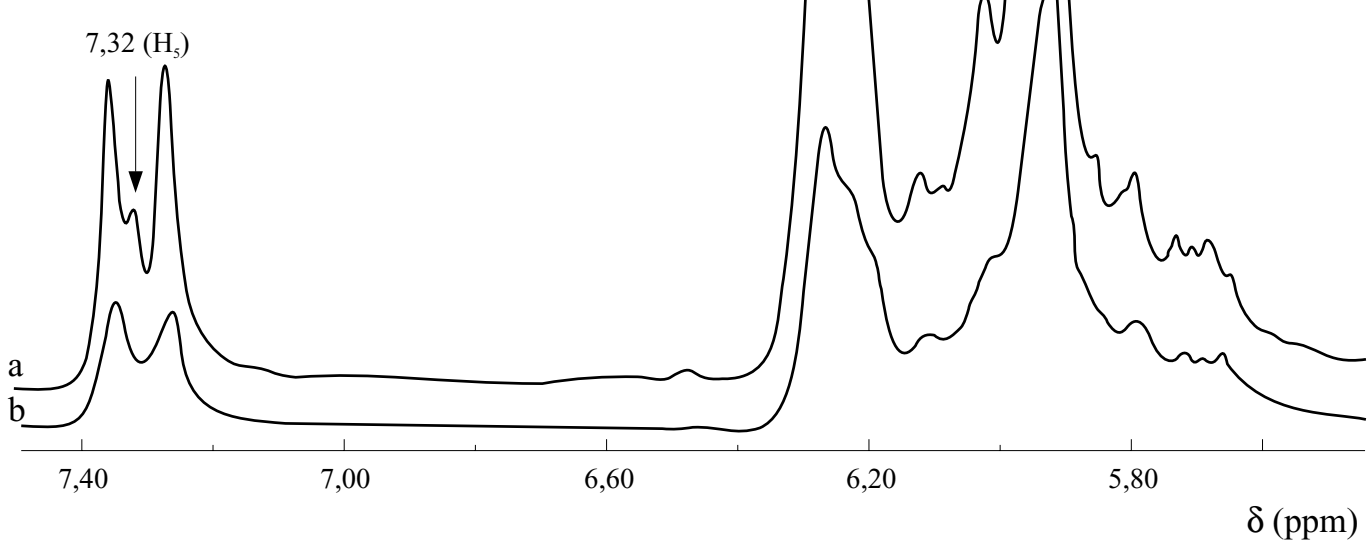

Figure 5. ${ }^{1} \mathrm{H}$ NMR spectrum of polyfurfuryl alcohol P3 (Table 1). (a) Spectrum of the original sample; (b) Spectrum of the sample after a treatment with $\mathrm{NaOH}$ solution $1 \mathrm{~N}$.

FA is hardly eliminated when the initial concentration of acid is low in relation to the monomer concentration and the reaction is stopped at low conversion of polymerization as in experiments of series $\mathrm{P} 3$. The presence of FA is observed in the ${ }^{1} \mathrm{H}$ NMR spectrum of this sample (Fig 5a). Difurfuryl ether (DFE) is also produced $^{[2]}$ with the experimental conditions of $\mathrm{P} 3$, but the ${ }^{1} \mathrm{H}$ NMR spectra of FA and DFE are indistinguishable and it is possible that the signals attributed to FA are actually due to a mixture of both substances.

The solution of P3 was washed twice with $1 \mathrm{M}$ $\mathrm{NaOH}$ and water, and a second ${ }^{1} \mathrm{H}$ NMR was recorded (Fig 5b). This resulted in the elimination of most of the FA.

\section{Photocross-linking.}

Gandini ${ }^{[12]}$ has remarked that labile hydrogen atoms on carbon atoms are the most likely candidates that initiate photodegradation or other degradation processes. The tertiary hydrogen atom of poly(2vinylfuran), for example, can be abstracted leading to a macroradical. The coupling of these radicals induces crosslinking.
PolyFA cross-links when exposed to the UV radiation of a Hg lamp. The mechanism probably involves the formation of macroradicals analogous to those obtained from poly(2-vinylfuran):

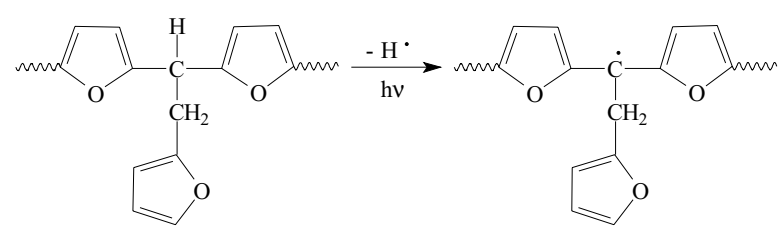

The major reaction pathways opened to the macroradicals should be:

- the attack on some olefin or dihydrofuran carbon atom of the polyunsaturated sequences

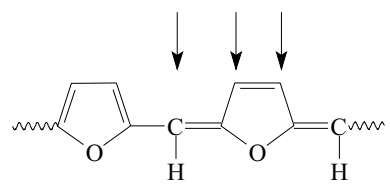


- the addition to monosubstituted furan rings<smiles>[R]CC(C)C(C)[C@H](C)c1ccc([R])o1</smiles>

The second pathway is probably more important. The polyunsaturated sequences are formed from the beginning of the reaction but their concentrations are very low. However, the proportion of monosubstituted furan ring is above $20 \%$ due to branching. It is well known that the addition of radicals to furan rings occurs predominantly at $\mathrm{C}_{2}$ or $\mathrm{C}_{5}$, with a kinetic preference for the unsubstituted position ${ }^{[13]}$.

PolyFA displayed properties of a negative photoresist. Two problems were observed when coating the $\mathrm{Cu}$ surface: the poor adherence of the polymer and some difficulties in the formation of films.

Adhesion was improved by introducing carbonyl group through chemical modification with maleic anhydride (MAN).

Figure 6 shows the comparison of the spectrum of polyFA (a) and the spectrum of the product from the reaction of polyFA with MAN (b). The presence of furan rings in both spectra is proved by the bands at 1600, 1150, 1020, 780 and $600 \mathrm{~cm}^{-1}$, whereas aliphatic segments give rise to the bands at 1420 and $1350 \mathrm{~cm}^{-1}[2,14]$.

Two important feature are observed in spectrum b: I) an increment of the relative intensity of the car- bonyl signal at $1700 \mathrm{~cm}^{-1}$ with respect to the signal at $1010 \mathrm{~cm}^{-1}$ (ring breathing ${ }^{[14]}$ ) and II) the occurrence of two signals at 1727 and $1767 \mathrm{~cm}^{-1}$, separated by $40 \mathrm{~cm}^{-1}$, which are characteristic of the carboxylic anhydrides ${ }^{[15]}$. Both feature point out the formation of the Diels-Alder adduct. MAN moiety contributes to the relative increase of the carbonyl signals by carboxylic anhydrides groups and by the carboxylic acid yielded from the hydrolysis of some anhydride groups.

The Mn of the adducted polyFA increases markedly when compared to the original polymer (from 3500 to $8200 \mathrm{~g} / \mathrm{mol}$ ).

Films of the adducted polyFa over glass have good performance as photoprinting material ${ }^{[16]}$ and films over silicon produces periodic interference patterns using ultraviolet laser ${ }^{[17]}$.

Films formation were improved by adding an acrylic copolymer composed of methyl methacrylate, butyl methacrylate and methacrylic acid (molar composition 42:49:9).

The modified resin was successfully tested as photoresist by making circuits on copper-coated boards. High quality images were developed on the copper surface. The photoresist was also tested on flat Si to

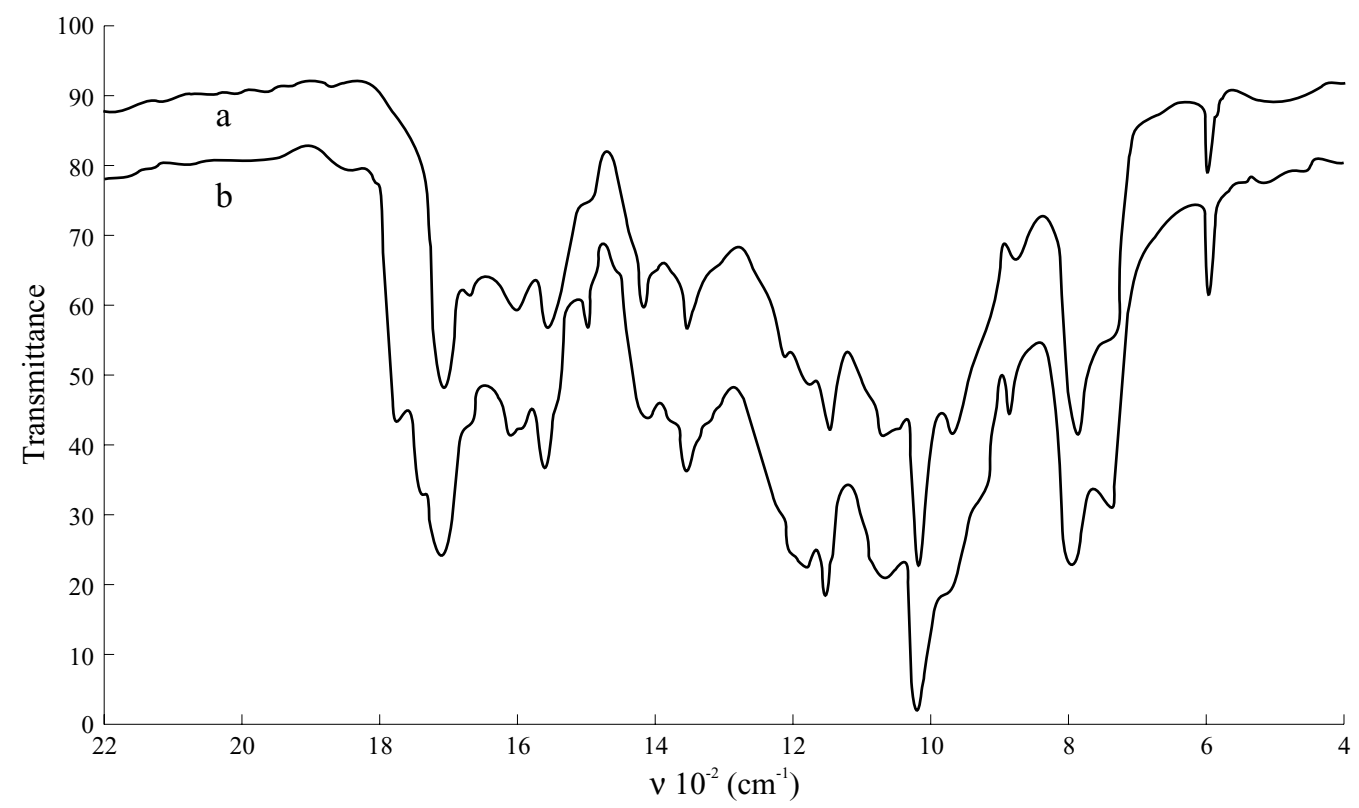

Figure 6. IR spectra of: (a) PolyFA; (b) PolyFA after the reaction with maleic anhydride 
make circuits. Good images were developed again and measurements concerning resolution will be performed.

The addition of 3,3'-diethyloxotiocianine iodide or the substitution of the acrylic copolymer by a polyalkenylfuran or a polyvinylphenylene also produces resists with good performance.

\section{Conclusion.}

PolyFA displays a tendency to retain the acids employed as catalysts hindering neutralization. Poorly neutralized polymers cross-link in solid state and exposed to air but not in solution.

The UV light initiates the reticulation of polyFA. The process is slow because the quantity of polyconjugated sequences in the polymer is relatively low. The presence of some acid accompanying the polymer film also induces reticulation. The combination of the two factors allows the development of images in lithographic processes.

\section{References}

1. Gandini, A. - Adv. Polymer Sci. 25, 47 (1977)

2. Gonzalez, R.; Martinez, R.; Ortiz, P. - Makromol. Chem. 193, 1 (1992)

3. Gonzalez, R.; Martinez, R.; Ortiz, P. - Makromol. Chem., Rapid Commun. 13, 517 (1992)

4. Gandini, A. - in "Encyclopedia of Polymer Science and Engineering", ed. H.F. Mark, Bikales, N.M.;
Overberger C.G.; Menges, G., Willey, New York, Vol. 7, p. 454 (1986).

5. Choura, M.; Belgacem, N.M.; Gandini, A. - Macromolecules, 29, 3839 (1996).

6. Lago, R.; Ortiz, P. J.; Martínez, R. - Acta Polym. 42, 403 (1991)

7. Gandini, A.; Martínez, R. - Rev. Cienc. Quím. 16: 275 (1985)

8. Martinez, R.; Alvarez, R.; Gandini, A. - Acta Polym. 40, 613 (1989)

9. Maciel, G. E.; Chuang, I. S.; Meyers, G. E.; Macromolecules 17, 1087 (1984).

10. Fawcett A. H; Dadamba W. - Makromol. Chem. 183, 2799 (1982).

11. Principe M.; Ortiz P.; Martínez R. - Polym. Int., 48, 637 (1999).

12. Gandini A.; Belgacem, N.M. - Progress in Polymer Science (1996)

13. Gandini A.; Rieumont, J. - Tetrahedron Lett. 25, 2101 (1976).

14. Katritsky, A. R. - "Physical Methods in Heterocyclic Chemistry", vol. 2, Academic Press Inc., New York (1956).

15. Marquarat, F. - J. Chem. Soc. B, 1242 (1966).

16. Sthel, M; Rieumont, J.; Martinez, R. - Polymer Testing 18(1), 47 (1999).

17. Sthel, M; Rieumont, J.; Martinez, R.. - Appl. Polymer Sci., 71, 1749(1999).

Recebido: 11/03/99

Aprovado: 14/01/00 